\title{
Mental health stigma among Middle Eastern adolescents: a protocol for a systematic review
}

\begin{abstract}
Introduction: Although many epidemiological and interventional investigations regarding improving mental health among youth in the Middle East have been performed, there is no systematic review specifically analyzing the studies on stigma among adolescents with mental disorders (A-MDs) across all Middle East countries despite their common historical roots, similar cultural backgrounds, and recent widespread problems in the area. Purpose: We will aim to systematically review and analyze the English-language studies on stigma among A-MDs in the Middle East published between 2000 and 2019. Method: The Web of Knowledge, Science Direct, PsycINFO and MEDLINE are electronic databases that will be included in this study. Furthermore, the bibliography lists of eligible articles will be manually searched for additional included articles. Descriptive statistics will be represented by mean score and standard deviation for continuous data and number/percentage for categorical data. Discussion: This study may act as a resource for future studies attempting to assess and/or reduce stigma among children and adolescents with mental health issues specifically in the Middle East. Implications: It is important for health care providers, including nursing professional, to improve their knowledge and vision towards stigma in their community. The outcomes of this study can be a shortcut reaching this information.
\end{abstract}

Keyword: Adolescents; Eastern Mediterranean region; Mental disorder; Mental health Stigma; Middle East region, Stigma 